\title{
Supporting Information Attenuation of abnormal scarring using spherical nucleic acids targeting transforming growth factor beta 1
}

Adam Ponedal ${ }^{\dagger 1,2}$, Shengshuang Zhu ${ }^{\dagger 2,3}$, Anthony J. Sprangers ${ }^{\dagger 2,4}$, Xiao-Qi Wang ${ }^{5}$, David C.

Yeo $^{6}$, Daniel C. S. Lio ${ }^{6}$, Mengjia Zheng ${ }^{6}$, Matthew Capek ${ }^{2}$, Suguna Narayan², Brian Meckes ${ }^{2,7}$,

Amy S. Paller*5, Chenjie Xu*8 ${ }^{*}$, Chad A. Mirkin*1,2,3,4

${ }^{1}$ Department of Chemical and Biological Engineering, Northwestern University, Evanston, IL, 60208

${ }^{2}$ International Institute of Nanotechnology, Northwestern University, Evanston, IL, 60208;

${ }^{3}$ Department of Materials Science and Engineering, Northwestern University, Evanston; IL, 60208

${ }^{4}$ Department of Biomedical Engineering, Northwestern University, Evanston, IL, 60208;

${ }^{5}$ Department of Dermatology, Northwestern University, Chicago, IL, 60611;

${ }^{6}$ School of Chemical and Biomedical Engineering, Nanyang Technological University, Singapore, 639798;

${ }^{7}$ Department of Biomedical Engineering, University of North Texas, Denton, TX, 76203;

${ }^{8}$ Department of Biomedical Engineering, City University of Hong Kong, Kowloon, Hong Kong SAR, China;

*To whom correspondence should be addressed (chadnano@ northwestern.edu, apaller@northwestern.edu, chenjie.xu@ cityu.edu.hk)

$\dagger$ These authors contributed equally 


\section{Supporting Figures \& Tables}
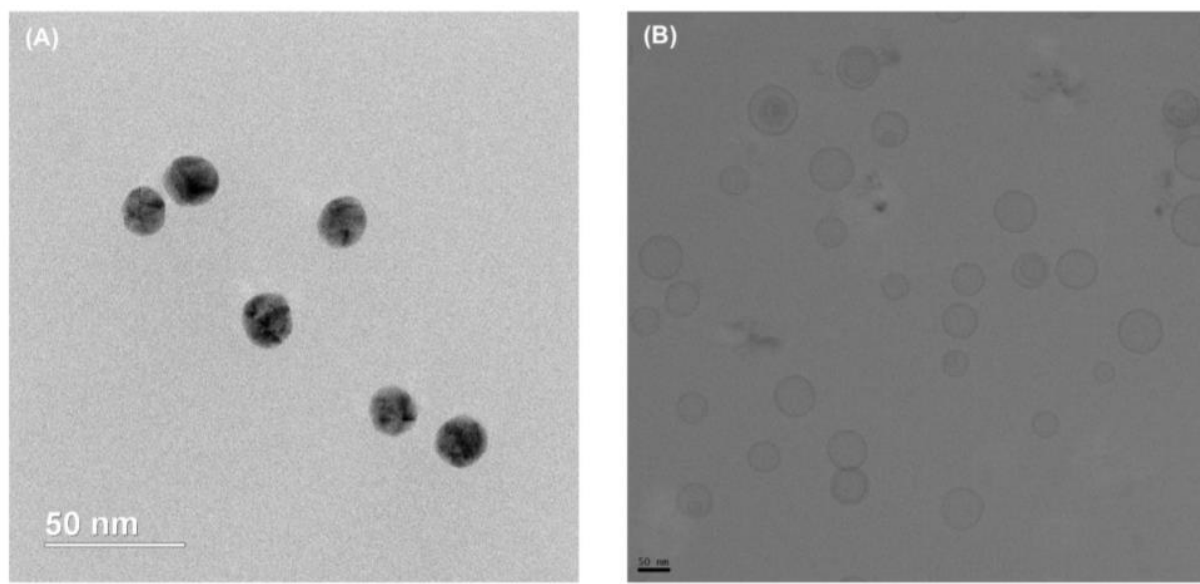

Figure S1. Transmission electron microscopy (TEM) images of AuSNAs and LSNAs. (A) Scanning transmission electron microscopy (STEM) image of AuSNAs. (B) Cryogenic TEM image of LSNAs.

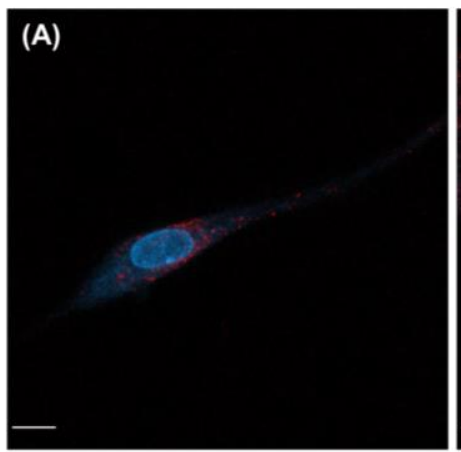

(D)

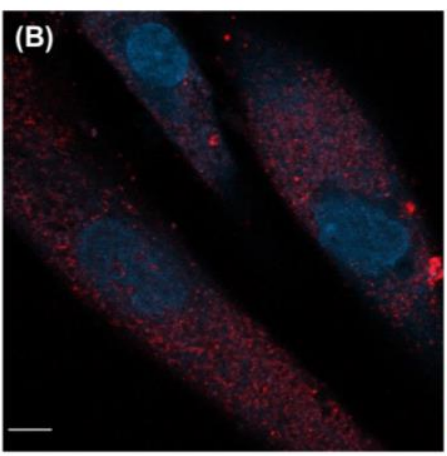

(E)

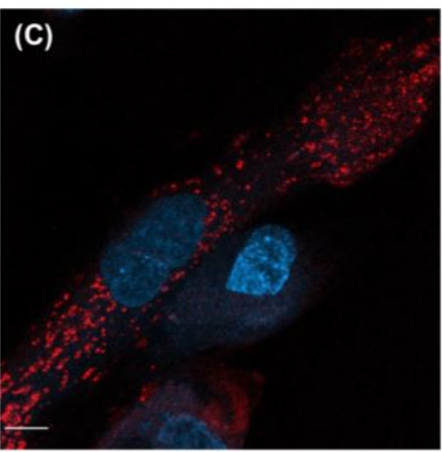

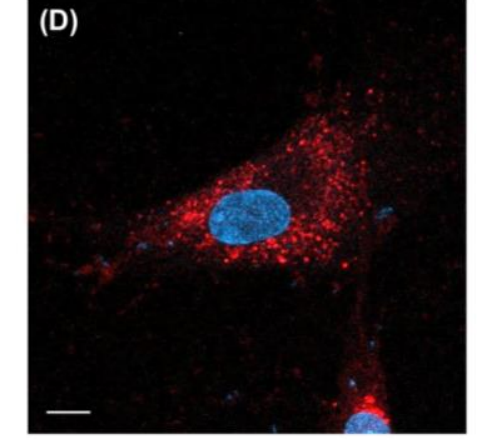

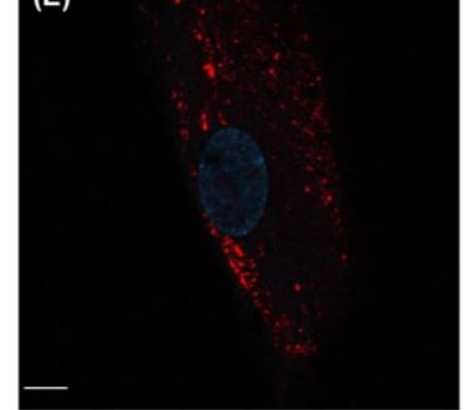

Figure S2. AuSNAs and LSNAs efficiently enter both rabbit and human fibroblasts. Confocal microscopy shows internalization of dye-labelled LSNAs in (A) Rab9 cells, (B) HSF cells, and (C) KF cells, and dye-labelled AuSNAs in (D) HSF cells and (E) KF cells. Scale bars represents $10 \mu \mathrm{m}$. 


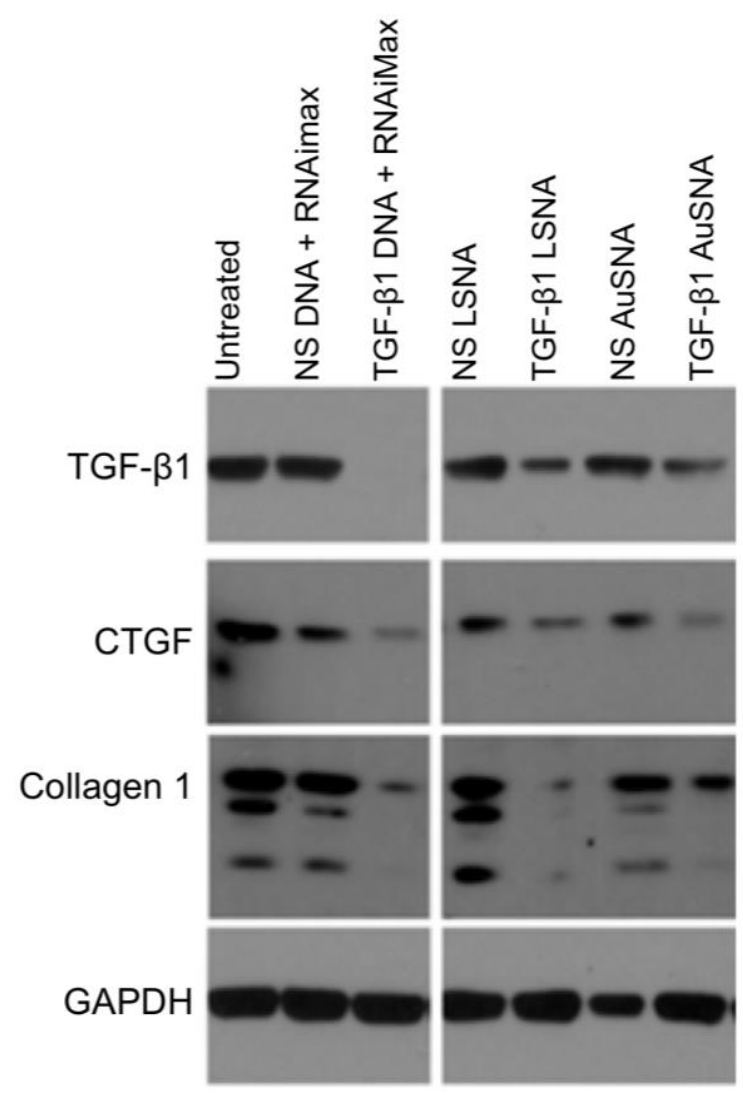

Figure S3. Knockdown of TGF- $\beta 1$ by SNAs in vitro results in reduced expression of proteins downstream in the TGF- $\beta 1$ signaling pathway. Western blot showing a decrease in CTGF and collagen 1 protein expression in patient-derived keloid fibroblasts, corresponding to the SNAmediated knockdown TGF- $\beta 1$. 


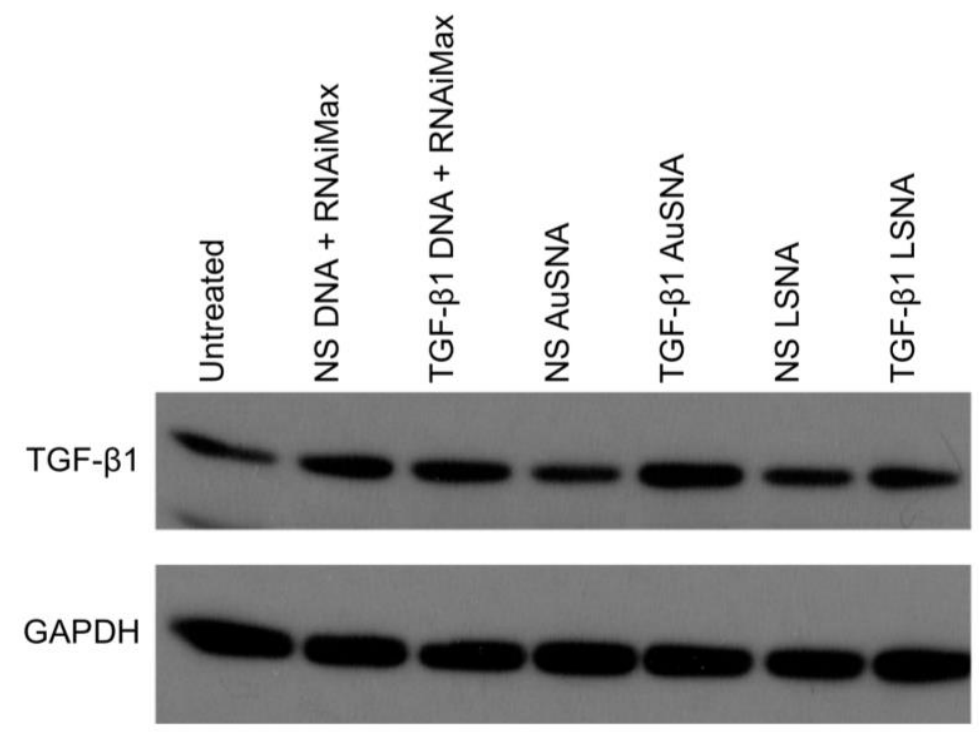

Figure S4. TGF- $\beta 1$-targeting antisense sequence and SNAs do not cause protein knockdown in mouse fibroblasts. Western blot showing that our TGF- $\beta 1$-targeting sequence and SNAs, homologous to only rabbit and human transcripts, do not reduce the protein's expression in mouse fibroblasts (NIH3T3). 


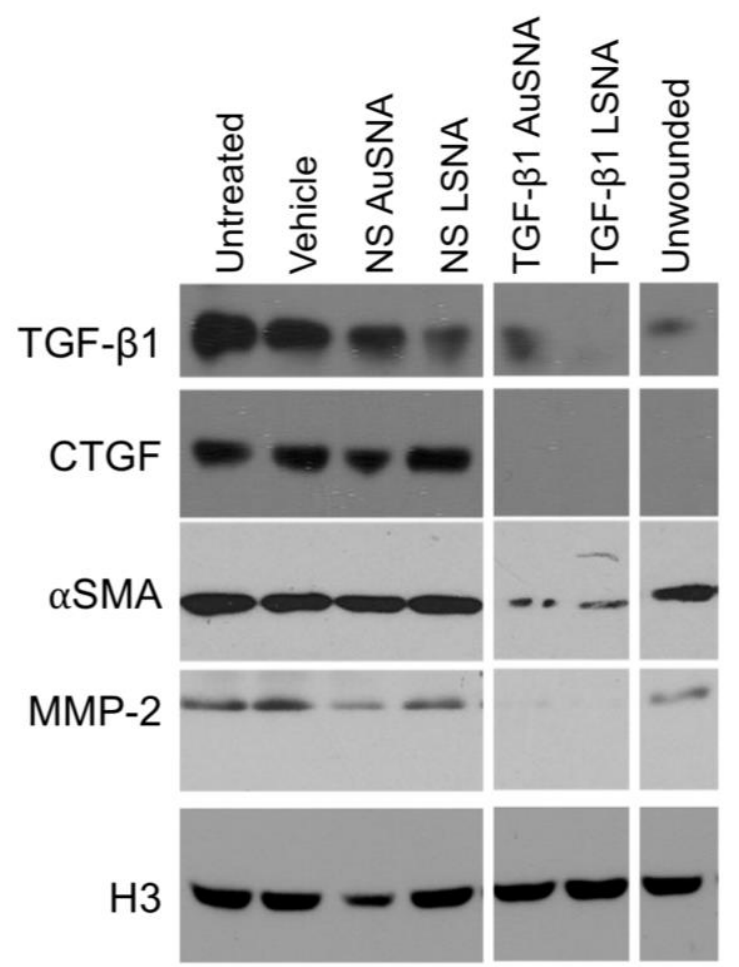

Figure S5. Knockdown of TGF- $\beta 1$ by SNAs in vivo results in reduced expression of proteins downstream in the TGF- $\beta 1$ signaling pathway. Western blot showing a decrease in CTGF, $\alpha \mathrm{SMA}$, and MMP-2 protein expression in rabbits, corresponding to the SNA-mediated knockdown TGF- $\beta 1$. We note that the ratio between $\alpha$ SMA and TGF- $\beta 1$ is different between the unscarred and unwounded groups. We attribute this difference to the complex nature of the aSMA expression pathway which, although known to be affected during abnormal scarring, ${ }^{1}$ is regulated by a complex and incompletely understood signaling network. . $3^{2}$

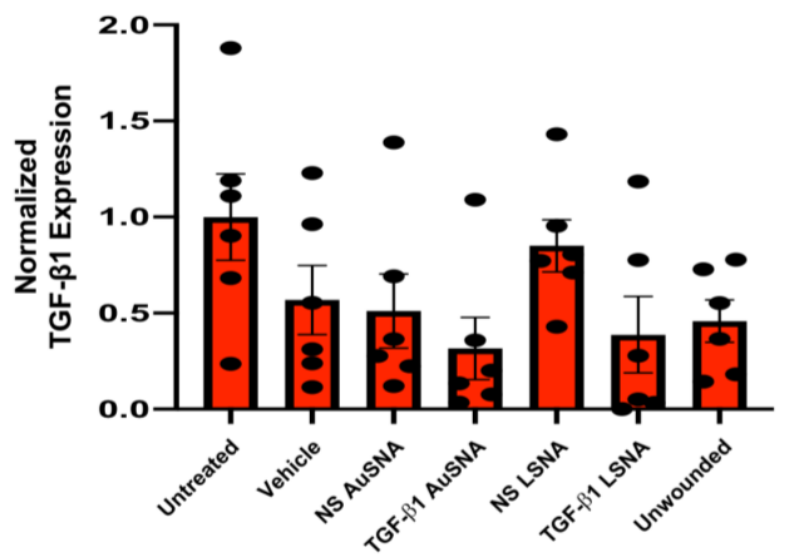

Figure S6. Average TGF- $\beta 1$ expression level, as quantified by densitometry of Western blot protein analysis with individual data points shown. 


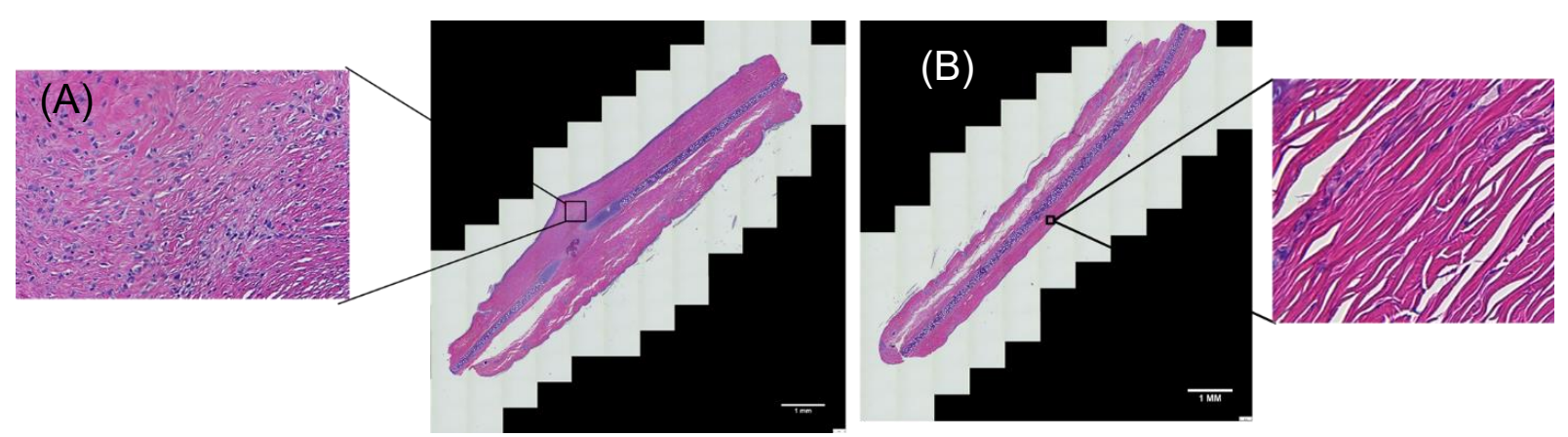

Figure S7. Representative images of H\&E-stained skin sections from scarred and healthy skin. (A) Scarred section with zoomed-in image on the left. (B) Healthy skin section with zoomed-in image on the right. Both of the zoomed-in images capture different regions of the dermis, albeit on different sides of the cartilage of the ear.

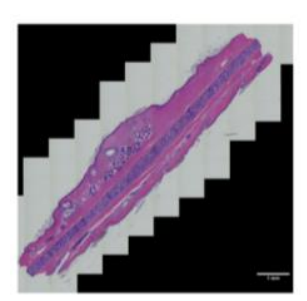

Untreated

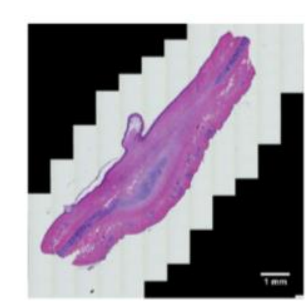

Vehicle

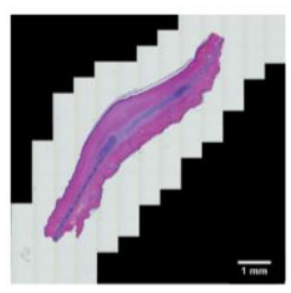

NS AUSNA

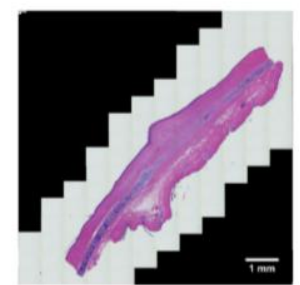

NS LSNA

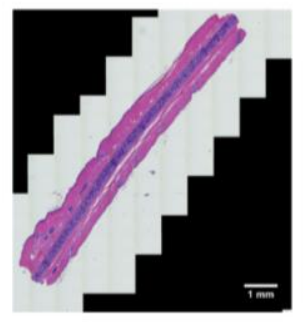

Unwounded

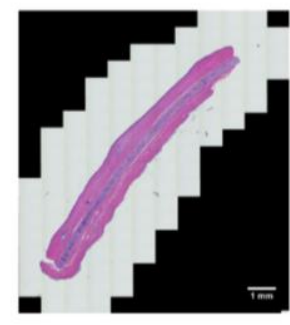

TGF-B1 AuSNA

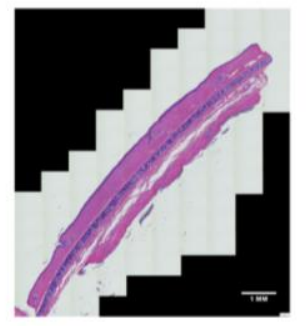

TGF- $\beta 1$ LSNA

Figure S8. Representative images of H\&E-stained skin sections for different treatment conditions. The images are oriented such that the scarred region is on top. 


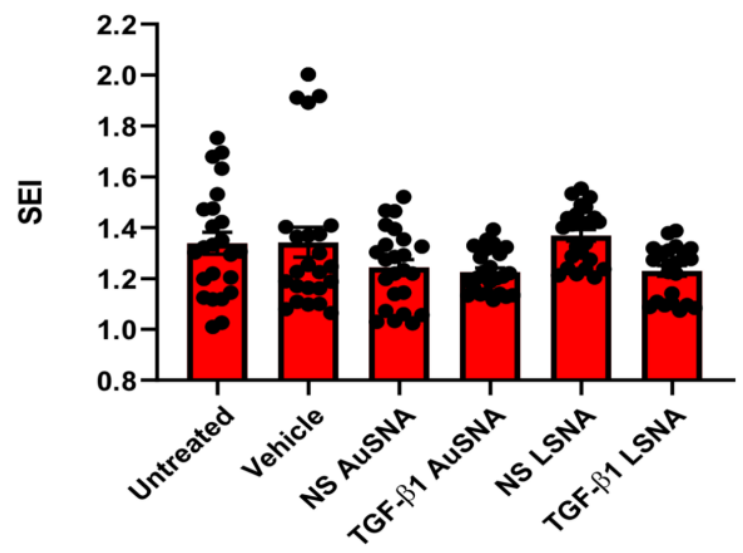

Figure S9. Composite SEI of different treatment groups with individual data points shown.

Table S1. Oligonucleotide sequences used in this study. Plus (+) sign indicates incorporation of locked nucleic acid.

\begin{tabular}{|l|l|}
\hline \multicolumn{1}{|c|}{ Name } & \multicolumn{1}{c|}{ Sequence } \\
\hline TGF- $\beta 1$ Antisense Thiol & GCC +AGC AGC CGG TTG C+TG +AG-(Spacer18) ${ }_{2}$-SH \\
\hline TGF- $\beta 1$ Antisense Tocopherol & (Tocopherol)-(Spacer18) ${ }_{2}$-GCC +AGC AGC CGG TTG C+TG +AG \\
\hline Nonsense Sequence Thiol & GTU+T TCA CCA CCC A+AT +TC-(Spacer18)2-SH \\
\hline Nonsense Sequence Tocopherol & (Tocopherol)-(Spacer18)2-GT+T TCA CCA CCC A+AT +TC \\
\hline $\begin{array}{l}\text { TGF- } \beta 1 \text { Antisense Thiol with Cyanine3 } \\
\text { (for cell uptake studies) }\end{array}$ & GCC AGC-(Cyanine3)-AGC CGG TTG CTG AG-(Spacer18)2-SH \\
\hline $\begin{array}{l}\text { TGF- } \beta 1 \text { Antisense Tocopherol with Cyanine3 } \\
\text { for cell uptake studies) }\end{array}$ & (Tocopherol)-(Spacer18) 2 -GCC AGC AGC CGG TT-(Cyanine3)-G CTG AG \\
\hline
\end{tabular}

Table S2. Zeta potential of SNAs and nanoparticles used to make them.

\begin{tabular}{|l|c|}
\hline \multicolumn{1}{|c|}{ Particles } & Zeta Potential $(\mathbf{m V})$ \\
\hline AuNPs & $-12.5 \pm 1.7$ \\
\hline AuSNAs & $-8.8 \pm 0.5$ \\
\hline Liposomes & $-1.24 \pm 1.22$ \\
\hline LSNAs & $-11.6 \pm 0.8$ \\
\hline
\end{tabular}


Table S3. $p$-values of statistical comparisons of TGF- $\beta 1$ expression and Scar Elevation Index (SEI).

\begin{tabular}{|l|c|c|}
\hline \multicolumn{1}{|c|}{ Comparison } & TGF- $\boldsymbol{\beta}$ Expression & SEI \\
\hline Untreated vs. Vehicle & not significant* & not significant \\
\hline Untreated vs. NS AuSNA & not significant & not significant \\
\hline Untreated vs. TGF- $\beta 1$ AuSNA & 0.0094 & 0.0243 \\
\hline Untreated vs. NS LSNA & not significant & not significant \\
\hline Untreated vs. TGF- $\beta 1$ LSNA & 0.0189 & 0.0308 \\
\hline Untreated vs. Unwounded & 0.0363 & N/A \\
\hline Vehicle vs. TGF- $\beta 1$ AuSNA & not significant & 0.0206 \\
\hline NS AuSNA vs. TGF- $\beta 1$ AuSNA & not significant & not significant \\
\hline Vehicle vs. TGF- $\beta 1$ LSNA & not significant & 0.0263 \\
\hline NS LSNA vs. TGF- $\beta 1$ LSNA & not significant & 0.0062 \\
\hline
\end{tabular}

*Significance defined as $p<0.05$

\section{REFERENCES}

1. Kopp, J.; Preis, E.; Said, H.; Hafemann, B.; Wickert, L.; Gressner, A. M.; Pallua, N.; Dooley, S., Abrogation of Transforming Growth Factor- $\beta$ Signaling by Smad7 Inhibits Collagen Gel Contraction of Human Dermal Fibroblasts. J. Biol. Chem. 2005, 280, 21570-21576.

2. Shinde, A. V.; Humeres, C.; Frangogiannis, N. G., The Role of A-Smooth Muscle Actin in Fibroblast-Mediated Matrix Contraction and Remodeling. Biochim. Biophys. Acta, Mol. Basis Dis. 2017, 1863, 298-309.

3. Secchiero, P.; Rimondi, E.; Di Iasio, M. G.; Voltan, R.; Gonelli, A.; Zauli, G., Activation of the P53 Pathway Induces A-Smooth Muscle Actin Expression in Both Myeloid Leukemic Cells and Normal Macrophages. J. Cell. Phys. 2012, 227, 1829-1837. 